\title{
Cooperative OSIC System to Exploit the Leakage Power of MU-MIMO Beamforming based on Maximum SLR for 5G
}

\author{
Cebrail ÇiFTLiKLi, Musaab AL-OBAIDI, Mohammed FADHIL and Wael AL-OBAIDI
}

\begin{abstract}
This study investigated the crucial—but not welldiscussed-issues involved in designing beamforming for all receivers, subject to leakage power constraints. Our assumption is that all users use ordered successive interference cancellation (OSIC) detection when the channel state information (CSI) is available. The problem of interest is to find beamforming that can improve OSIC performance of a multi-user scheme without significantly increasing the complexity. This study considers the transceiver design for multi-user MIMO (MU-MIMO) communications, in which a single transmitter adopts beamforming to simultaneously transmit information at first time-slot. During the second time-slot, receivers cooperate to share specific results of OSIC detection in each user. We propose the maximum-likelihood (ML) to estimate the received symbols. The estimated symbols will be used in OSIC detection to detect interference symbols. Promising results show that our cooperative OSIC scheme of the MU-MIMO beamforming system based on maximum signal-to-leakage ratio (SLR) realizes the diversity order of OSIC. Also, by utilizing leakage power as a useful power and not just as an interference power, the performance of the proposed scheme over Rayleigh and Rician channels is significantly better than the performance of classical MU-MIMO beamforming system based on SLR at a high signalto-noise ratio (SNR).
\end{abstract} ML.

Index Terms - MU-MIMO, Beamforming, CSI, SLR, OSIC,

\section{INTRODUCTION}

$\mathrm{C}$ ommunication over MIMO channels has appealed to scientists because of its promising results and extraordinary performance. MIMO systems have the tremendous potential to achieve great throughput and high reliability in wireless communication systems [1]. Recently, especially since the discovery of the MIMO broadcast channel capacity, attention has shifted to the MU-MIMO wireless

This paper submitted in 12 Jan 2019.

Cebrail CiFTLiKLi, he joined Vocational College, Erciyes University, Kayseri, Turkey, as Professor where he is now principal (e-mail: cebrailc@erciyes.edu.tr).

Musaab AL-OBAIDI, he is Ph.D. student in Department of Electrical and Electronics Engineering, Erciyes University, Kayseri, Turkey (e-mail: musaabsami2000@yahoo.com).

Mohammed Fadhil, he is currently Ph.D. candidate at Universiti Kebangsaan Malaysia (UKM), Kuala Lumpur, Malaysia (e-mail: moh fadhil77@yahoo.com).

Wael AL-OBAIDI, he is M.Sc. student in Department of Electrical and Electronics Engineering, Erciyes University, Kayseri, Turkey (e-mail: wael.s.ismaeel@gmail.com). system [2]-[4], which is emerging wireless communication technology. It is highly expected that future $5 \mathrm{G}$ networks should achieve a 10 -fold increase in connection density, i.e., $10^{6}$ connections per square kilometers [5].

Beamforming scheme based on SLR technology enables the MU-MIMO system to provide good quality of service (QoS), thus absorbing more users and makes it promising to address the $5 \mathrm{G}$ requirement of massive connectivity. Specifically, in Internet of Things (IoT) scenario and massive machine-type communications (mMTC) scenario, which are considered as typical $5 \mathrm{G}$ application scenarios. In these scenarios, users may be low-cost sensors deployed in a small area, where both lineof-sight (LOS) and non-line-of-sight (NLOS) exist, which can be better modeled by the Rician fading channel. $4 \mathrm{G}$ and $5 \mathrm{G}$ cellular systems rely on MU-MIMO transmitters, which use linear precoding [6]-[9]. Although linear precoding is incapable of achieving the channel capacity in regions of MIMO broadcasts, it is an attractive choice because of its simplicity. In MU-MIMO systems, several users with an antenna array employ identical frequency bands and time slots to communicate with the base station (BS). Therefore, these systems are susceptible to co-channel interference (CCI) [10], which can decline the overall capacity of MU-MIMO systems. In [11], the system performance with regards to the Alamouti scheme undergoes both analysis and evaluation with block diagonalization precoding applied when there is the presence of CSI.

For optimized performance, it is extremely important to suppress CCI [12]. Although the transmitter can coordinate transmission from all of its antenna elements, users cannot coordinate with one another. Overcoming this inability of the user is the major challenge in MU-MIMO systems [13]. To ensure the complexity is lower at the receiving end, transmitter optimization must be addressed. The most commonly used techniques for transmission optimization include CSI at the transmission side to deal with the CCI. This technique will definitely reduce overhead due to channel feedback.

Numerous works have suggested such schemes for perfectly cancelling the CCI for each receiver [14]-[17]. Although these schemes lead to excellent performance, they tend to restrict the system configuration because it requires using a specific number of antennas. This approach also results in obvious system capacity cutbacks as the useful power of the received signals is significantly reduced at a low SNR [18]. 


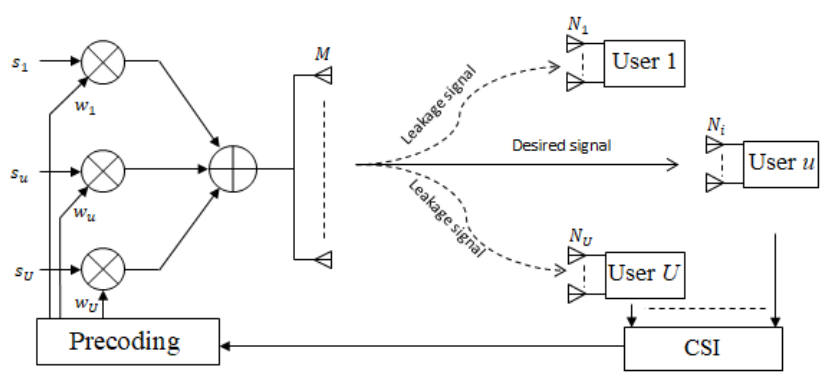

Fig. 1. Block diagram of the MU-MIMO beamforming system.

Other approaches rely on using algorithms to boost or maximize the useful power of received signals. However, these algorithms do not attempt to reduce multi-user interference. Hence, the systems based on these algorithms are also not perfect owing to their susceptibility to interference at high SNRs [19]. Broadly, all these systems either attempt to maximize the output of signal-to-interference-plus-noise ratio (SINR) or minimize the transmission power while satisfying the SINR targets for all the users. Owing to the coupled nature of both signals and interference, most of the solutions are typically iterative. Algorithm-based systems also lose the same power to keep these systems simple and uncomplicated. We actually are crawling from simplicity to little complexity in the BS and, in some cases, shifting gradually from little complexity to clear complexity.

This study proposes a novel MU-MIMO downlink scheme. This study considers an alternative approach based on the concept of SLR to design transmits beamforming vectors and uses OSIC [20] in each user to detect its own signal and then detect other users' signals. Our suggested system encourages cooperation between users to share the interference signal so that the benefit of leakage power can be realized. In our proposed system, the complexity in the BS will be reduced by giving very few tasks to the users to support the desired signal by reusing the leakage signal. In the MU-MIMO beamforming system based on SLR, the design procedure for precoding weight needs to use constantly of proportionality, which is chosen to normalize the norm of the precoding to unity [10]. Comparing this solution to the proposed scheme; there is no need to do this step in BS. Also, in our proposed scheme, a cooperative SLR transmission scheme did not use coding in the transmission process and thus did not use the decoding and recoding in the user. Extensive simulations in the MU-MIMO system have been carried out over Rayleigh (in the NLOS environment) and Rician fading channels (in the LOS environment).

The simulation results demonstrate that the proposed scheme outperforms classical schemes at high SNRs. Furthermore, it can significantly reduce the BS complexity. The rest of the paper is organized as follows. The system model is introduced in Section II. In Section III, we review the SLR precoding. Section IV provides proposed scheme. In Section $\mathrm{V}$ we provide and discuss the simulation. In section VI concludes the paper. Finally, in section IX we provide the contribution of this paper. The superscripts $(\cdot)^{\mathrm{T}},(\cdot)^{*},(\cdot)^{\mathrm{H}}$, and $(\hat{\cdot})$ denote transpose, complex conjugate, Hermitian operations, and noisy version of $(\cdot)$, respectively.

\section{SYSTEM MODEL}

We considered an environment of downlink MU-MIMO broadcast channel consisting of $U$ geographically sparse mobile stations communicating with the BS that has $M$ antennas. In such an environment system, each user is independent. Let user $i$ employing $N_{i}$ antennas. This user will receive its own signal, as shown in Fig. 1. This user will receive its own signal, while the other users will receive the leakage signal of user $i$. We define $N_{T}=\sum_{u=1}^{U} N_{u}$ as the total number of users' antennas for $u=\{1, \ldots, U\}$. For a typical wireless communication network, we imagine that $N_{T} \geq M$ with the independent channels of flat fading. Suppose that the intended message signal for user $i$ is the scalar $s_{i}$ [10]. Therefore, the transmitted symbol vector to $U$ users is:

$$
S=\left[s_{1}, \ldots, s_{U}\right]^{T}
$$

In the precoding step, the matrix of precoding is denoted as follows:

$$
W=\left[w_{1}, \ldots, w_{U}\right]
$$

where $w_{i} \in C^{M \times 1}$ is the joint precoding vector (beamforming coefficients) for the user $i$. After the joint precoding step, the symbol is multiplied by beamforming vector and then the transmitted vector will be:

$$
X=\sum_{u=1}^{U} w_{u} s_{u}=W S
$$

It is assumed that signals $W S \in C^{M \times 1}$ are broadcast over the channels denoted as:

$$
H=\left[H_{1}^{T}, \ldots, H_{U}^{T}\right]^{T}
$$

where $H_{i} \in C^{N_{i} \times M}$ describes the channel coefficients between the $N_{i}$ receiver antenna at the user $i$ and BS antennas as follows:

$$
H_{i}=\left[\begin{array}{ccc}
h_{i}^{(1,1)} & \ldots & h_{i}^{(1, M)} \\
\vdots & \ddots & \vdots \\
h_{i}^{\left(N_{U}, 1\right)} & \ldots & h_{i}^{\left(N_{U}, M\right)}
\end{array}\right]
$$

where $h_{i}^{(n, m)}$ denotes the channel coefficient between the BS, which has the $m$ transmitter antenna, and the user $i$, which has the $n$ receiver array antenna. Thus, the received signals at the receivers' antennas are:

$$
\boldsymbol{y}=\left[y_{1}{ }^{T}, \ldots, y_{U}^{T}\right]^{T}=H W S+n o
$$

where $y_{i} \in C^{N_{i} \times 1}$ represents the signal received at the recipient $i$, while that for the additive noise is denoted by $n o \in C^{U N_{i} \times 1}$.

When each user has been carefully considered separately, we will find the received signal at a recipient $i$ as:

$$
\begin{aligned}
& y_{i}=H_{i} \sum_{u=1}^{U} w_{u} s_{u}+n o_{i} \\
= & H_{i} w_{i} s_{i}+H_{i} \sum_{u=1, u \neq i}^{U} w_{u} s_{u}+n o_{i}
\end{aligned}
$$


The $H_{i}$ vector has complex Gaussian variable components with unit variance and zero mean. Moreover, the components of the additive noise $n o_{i}$ have a distribution as $N\left(0, \sigma_{i}^{2}\right)$ and are temporarily and spatially white. To describe the proposed scheme clearly, the original SLR-based precoding scheme [10] is reviewed in the next section.

\section{SLR PRECODING}

The original SLR-based precoding scheme is reviewed in [10]. Recall that SLR is defined as the ratio of received signal power at the desired user to received signal power at the other terminals (the leakage power) plus noise power without considering receive matrices. This scheme computes the maximum beamforming precoding $\left(w_{i}^{o}\right)$ of each user from the maximum SLR of these users [10] as follows:

$$
S L R=\frac{\left\|H_{i} w_{i}\right\|^{2}}{\sum_{u=1 . u \neq i}^{U}\left\|H_{u} w_{i}\right\|^{2}}
$$

Then

$$
w_{i}^{o}=\arg \max \frac{\left\|H_{i} w_{i}\right\|^{2}}{\sum_{u=1 . u \neq i}^{U}\left\|H_{u} w_{i}\right\|^{2}}
$$

where $\left\|H_{i} w_{i}\right\|^{2}$ represents the required signal power of user $i$, while $\sum_{u=1 . u \neq i}^{U}\left\|H_{u} w_{i}\right\|^{2}$ represents the total leakage power from the total power of user $i$ as an interference to the other users. By substituting $\widetilde{H}_{i}=\sum_{u=1 . u \neq i}^{U} H_{u}$ into (8), we can obtain

$$
S L R=\frac{\left\|H_{i} w_{i}\right\|^{2}}{\left\|\widetilde{H}_{i} w_{i}\right\|^{2}}=\frac{w_{i}^{*} H_{i}^{*} H_{i} w_{i}}{w_{i}^{*} \widetilde{H}_{i}^{*} \widetilde{H}_{i} w_{i}}
$$

As in [10] we can solve (9) as

$$
\frac{w_{i}^{*} H_{i}^{*} H_{i} w_{i}}{w_{i}^{*} \widetilde{H}_{i}^{*} \widetilde{H}_{i} w_{i}} \leq \lambda_{\max }\left(H_{i}^{*} H_{i}, \widetilde{H}_{i}^{*} \widetilde{H}_{i}\right)
$$

where $\lambda_{\max }$ is the largest generalized eigenvalue. According to the SLR criterion, the precoding matrix $w_{i}$ is designed based on the following metric:

$$
w_{i}^{o} \propto \max \text { gen. eigenvector }\left(H_{i}^{*} H_{i}, \widetilde{H}_{i}^{*} \widetilde{H}_{i}\right)
$$

Depending on [10], the proportionality constant is chosen to normalize the norm of $w_{i}^{o}$ to unity. At user $i$, the maximumlikelihood detection scheme will be used to estimate $s_{i}$ from the received signal as follows [10]:

$$
\hat{s}_{i}=\frac{w_{i}^{*} H_{i}^{*}}{\left\|H_{i} w_{i}\right\|^{2}} y_{i}
$$

Then

$$
\hat{s}_{i}=s_{i}+\frac{w_{i}^{*} H_{i}^{*} \sum_{u=1 . u \neq i}^{U} H_{i} w_{u} s_{u}}{\left\|H_{i} w_{i}\right\|^{2}}+\frac{w_{i}^{*} H_{i}^{*}}{\left\|H_{i} w_{i}\right\|^{2}} n o_{i}
$$

\section{COOPERATIVE ALGORITHM}

Considerable limited network resources such as energy can be saved through cooperation, which also increases the reliability and quality of services. Quality and reliability are measured by parameters such as bit error rate and outage probability. Hence, cooperation can extend the coverage range and data throughput.

\section{Amplify-And-Forward (AAF)}

A simple form of wireless cooperative communication is the AAF method. In this method, the signal received by each relay is a noise signal. It suffers from attenuation. Therefore, the noisy version of the original signal needs to be amplified before it can be sent again by the partner or relay. In doing so, the noise in the signal is also amplified. At the destination, the information transmitted by the user and partner will be combined. Although the AAF method amplifies the signal, it also amplifies the noise. Laneman and Wornell [21] present and analyze the AAF method.

\section{Decode-And-Forward (DAF)}

This method involves two sub-steps. First, the partner decodes the user's information, and second, the decoded information is retransmitted to the destination. Therefore, there is no amplified noise in the sent signal. The DAF is the most preferred method in a relay. This method can only be meaningfully provided the relay can decode the original message completely. Sendonaris employed the DAF signaling in a simple code-division multiple access (CDMA) system with two users [22].

\section{PROPOSED SCHEME}

In this section, a novel MU-MIMO system is proposed aimed at fully benefiting from the transmission power to each user. In practice, in MU-MIMO beamforming based on maximum SLR system, the power leaked from one user to the others is different. Power control in BS is usually applied to design a beamforming coefficient for each user.

From the literature on SLR precoding described in Section III, we consider an appropriate combination of three algorithms.

\section{Precoding Design}

Based on [19], the drawback of [10] is that when each user has multiple data streams, the effective channel gain for each stream can be severely unbalanced. If power control or adaptive modulation and coding cannot be applied, then the overall error performance of each user will suffer a significant loss. Therefore, unlike the original SLR scheme in [10], which gives the solution in Equation (12) dependent on subject to $\left\|w_{u}\right\|^{2}=1$, we apply total transmission power constraints $\left(P_{u}\right)$ at the transmitter, which can be described as $E\left(\left\|w_{u} s_{u}\right\|^{2}\right) \leq$ $P_{u}$. The symbol $\mathrm{s}_{\mathrm{u}}$ satisfies the power constraint as $E_{u}=$ $E\left(\left|s_{u}\right|^{2}\right)=1$. Therefore, the same solution is chosen in Equation (12) but depends on [23]:

$$
\text { subject to }\left\|w_{u}\right\|^{2}=w_{u}{ }^{H} w_{u} \leq P_{u} / E_{u}
$$

Note that the norm of $w_{u}$ is irrelevant to the final solutions; in other words, the norm of $w_{u}$ can be forced to be any value to achieve the best value for $w_{u}$ under the power constraints. 


\section{OSIC Detection Algorithm}

Linear detection methods require a low complexity of hardware implementation. The OSIC method helps to improve performance without increasing complexity. In OSIC, based on the received signal at all the antennas, the symbols are selected to remove the effect from the others. To reduce the interference, the detected signal in each stage is subtracted from the received signal [20]. Suppose that the ML detection scheme will be used to estimate $s_{i}$ from the received signal. After estimation, the remaining signal in the first stage is formed by subtracting it from the received signal $y_{i}$. After estimation $s_{i}$, the remaining signal in the first stage is formed by subtracting it from the received signal as shown in Fig. 2, that is,

$$
\hat{y}_{i}=y_{i}-H_{i} s_{i}
$$

then the interference is successfully canceled in the course of estimating $s_{u}, u=1, \ldots, i-1, i+1, \ldots, U$. After estimating all $s_{u}$ in each user, all users cooperate with one another to share the interference signal only to benefit from the leakage power.

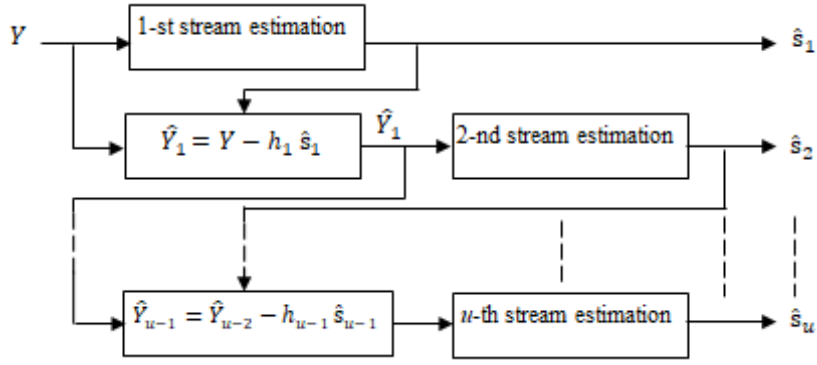

Fig.2 Illustration of OSIC-MU signal detection for $u$-th spatial streams of user 1

\section{Hybrid Relaying Model}

Our suggestion cooperative method is different from DAF as well as from AAF method. We produce a Hybrid Relaying model of DAF and AAF which does not depend on coding the signal in BS like DAF method, but it depends on the estimation of the leakage signal using OSIC (without decoding and recoding the leakage signal like DAF method) in the first user (or relay). Then the estimated leakage signals multiplies by control power parameter $(\beta)$ and it transmits to the second user (or destination) without increasing the effect of noise as in AAF method. In this way, the complexity of BS will reduce based on dispense of using coding technique in BS. Also, the decoding and recoding the leakage signal in the relay was excluded, to reduce the processing latency, thus reducing the complexity load in real-time detection in the relay and depend on signal estimation and leakage power in the user side. In the proposed scheme, at the first interval, using ML estimation and OSIC detection, each user differentiates and identifies its own signal (desired signal) from other user signals known as the leakage signal. At the second interval, the leakage signals for other users are transmitted to them. By using this scheme, we can benefit from leakage power and turn it into a productive power by using the combiner in each user to combine the desired signal detected by each user as its own signal with the leakage signal detected by other users as an interference signal. When the inter-user channel (the channel between users) is appropriate, the control power $(\beta)$ will be used for the interference signal cooperation. Simply put, to take advantage of the leaked signal, the signal leaked from the original signal will be reintroduced to its real destination. Total transmission power constraints exist at the transmitter, which can be described as $E\left(\left\|\beta_{i} s_{i}\right\|^{2}\right) \leq P_{i} . \beta_{i}$ is a constant to meet the total transmitted power constraint and is given as follows [20]:

$$
\beta=\sqrt{\frac{N_{T}}{\operatorname{Tr}\left(H^{-1}\left(H^{-1}\right)^{H}\right)}}
$$

Therefore, the transmitted signal from $u$-th to $i$-th user at the second time slot is:

$$
\hat{s}_{u-i-2 n d}=\beta * \hat{s}_{u-i-1 s t}
$$

where $\hat{s}_{u-i-1 s t}$ is the noisy version of the leakage signal $\left(s_{u-i-1 s t}\right)$ from the $i$-th user who is detected by the $u$-th user at the first time slot. The received signal at the second time slot in $i$-th user is given by

$$
y_{u-i-2 n d}=H_{u-i-2 n d} * \hat{s}_{u-i-2 n d}+n o_{i}
$$

where $H_{u-i-2 n d}$ is the inter-user channel between the user $u$ and user $i$. $n o_{i}$ is the AWGN in user $i$. In user $i$, maximum ratio combiner (MRC) will be used to combine the desired signal $\left(\hat{s}_{i}\right)$, which is detected as its own signal at the first time slot, with $\hat{s}_{u-i-2 n d}$. By using MRC scheme, the signal of $i$-th user will be

$$
\begin{aligned}
s_{i} & =\hat{s}_{i}+\sum_{u=1, u \neq i}^{U} \hat{s}_{u-i-2 n d} \\
& =\hat{s}_{i}+\sum_{u=1, u \neq i}^{U} \frac{H_{u-i-2 n d}^{*}}{\left\|H_{u-i-2 n d}\right\|^{2}} * y_{u-i-2 n d}
\end{aligned}
$$

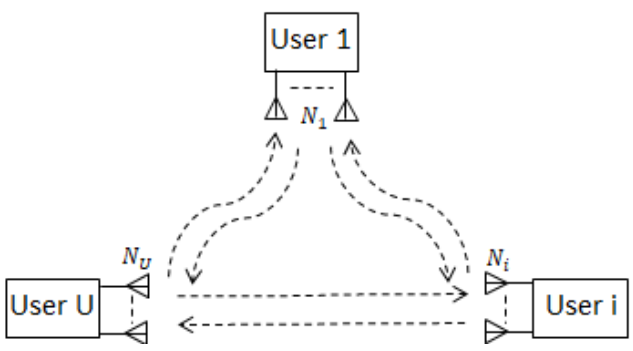

Fig. 3 Cooperative of OSIC-MU signal detection for $u$-th spatial streams of $u$-th user

\section{DOWNLINK CHANNEL MODEL}

Due to LOS propagation, the strongest propagation component of MIMO channel corresponds to the deterministic component (also referred to as specular components). On the other hand, all the other components are random components (due to NLOS also referred to as scattering components) [20]. 


\section{DOWNLINK CHANNEL MODEL}

Due to LOS propagation, the strongest propagation component of MIMO channel corresponds to the deterministic component (also referred to as specular components). On the other hand, all the other components are random components (due to NLOS also referred to as scattering components) [20].

The broadcast channel distribution has been following the Rayleigh channel distribution which is Gaussian distribution with a variance of $\sigma^{2}$ and zero mean. That means there is no component of $\operatorname{LOS}(K=0): \sigma=\sqrt{\frac{1}{K+1}}$. On the other hand, when there is any component of LOS (For $K>0$ ) the broadcast channel distribution has been following the Gaussian distribution with a variance of $\sigma^{2}$ and mean of $q$ or Rician distribution when $K$ increases as: $q=\sqrt{\frac{K}{K+1}}, \sigma=$ $\sqrt{\frac{1}{K+1}}$. Therefore, in this work, the channel matrix of MIMO system is described as [24]:

$$
H=\sqrt{\frac{K}{K+1}} H_{d}+\sqrt{\frac{1}{K+1}} H_{r}
$$

where $H_{d}$ is representing the component of the normalized deterministic channel matrix, while $H_{r}$ is representing the component of random channel matrix, with $\left\|H_{d}\right\|^{2}=N_{T} M$, $E\left\{\left|\left[H_{r}\right]_{i, j}\right|^{2}\right\}=1, i=1: N_{T}, j=1: M[24]$. While $K$ is known as a factor of the Rician channel which is the relation between the component of the specular power $c^{2}$ and the component of scattering power $2 \sigma^{2}$, displayed as [20]:

$$
K=\frac{\left\|H_{d}\right\|^{2}}{E\left\{\left|\left[H_{r}\right]_{i, j}\right|^{2}\right\}}=\frac{c^{2}}{2 \sigma^{2}}
$$

\section{SIMULATION RESULTS AND EVALUATION}

In this part, it was evaluated the signal-to-noise ratio (SNR) against the bit error rate (BER) as a scale of precoding efficiency. A typical MU-MIMO scheme is imitated to estimate the performance of the suggested MU-MIMO beamforming precoding scheme over the Rayleigh fading channel as a conventional channel in the first time slot (downlink channel) and Rician fading channel as a realistic channel in the second time slot ( inter-user channel). For random Rayleigh and Rician fading channel case, the samples of these parameters are set up to 1000 with elements generated as zero-mean for Rayleigh fading channel while $m$-mean for Rician fading channel and unit-variance independent and identically distributed (i.i.d) complex Gaussian random variables. We simulate MU-MIMO system, where the average BER is taken of SLR-based precoding approach at BS while the receiver was using a ML with OSIC detection approach.

BS transmits by $M$ antennas to each user over the noisy channel and flat fading channel while each user has employed $N_{u}$ antennas to receive the signal. QPSK signal constellation has been used as a broadcast modulation in all simulations and the results are averaged through several channel investigations. For all receivers, the noise variance per receive antenna is supposed to be equal, $\sigma_{1}^{2}=\ldots=\sigma_{U}^{2}=\sigma^{2}$. As for the power constraints, $P_{u}=P_{i}=1$ is considered. The typical values and simulation parameters are presented in Table 1 .

Table 1.

Typical values and simulation parameters.

\begin{tabular}{|l|l|}
\hline \multicolumn{1}{|c|}{ Parameters } & \multicolumn{1}{c|}{ Definition } \\
\hline Channel type & Rayleigh and Rician \\
\hline SNR of inter user channel & $15 \mathrm{~dB}$ \\
\hline Number of users $(U)$ & 3 \\
\hline Number of antenna for BS $(M)$ & 6 \\
\hline Number of antenna in each user $(N i)$ & 3 \\
\hline Rician channel factor $(K)$ & $10-15 \mathrm{~dB}$ \\
\hline Beta $(\beta)$ & $0.075-0.15$ \\
\hline
\end{tabular}

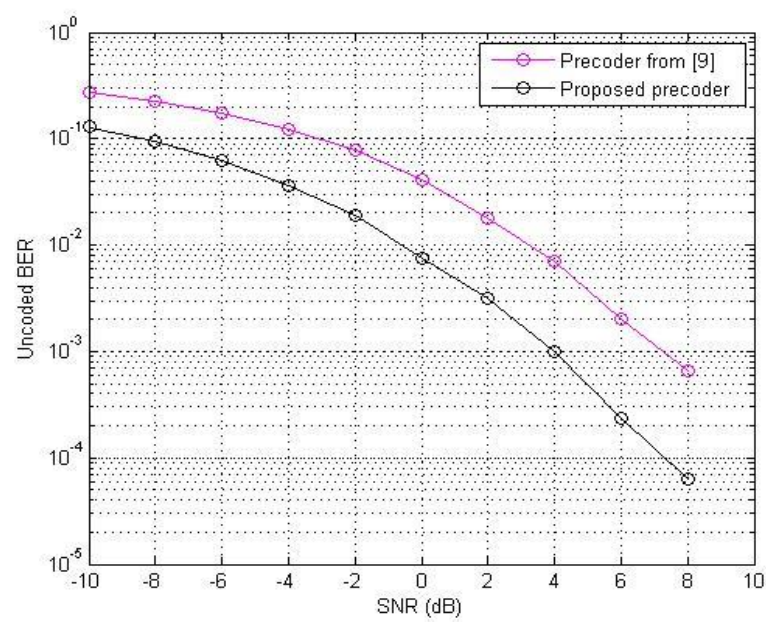

Fig.4 MU-MIMO system for $M=6, U=3, N i=3$.

Fig. 4 illustrates the comparison of the classical MU-MIMO beamforming system based on SLR and the proposed precoder. While the classical MU-MIMO beamforming system based on SLR, which maximizes the useful power of users and neglects the MU interference fails in a multi-user environment, to pass the proposed precoder, the proposed precoder provides an acceptable BER. For example in Figure 5 , the classical MU-MIMO beamforming system based on SLR achieves $10^{-3}$ uncoded BER at a SNR of $7 \mathrm{~dB}$ while the proposed precoder maintains an acceptable $10^{-3}$ uncoded BER for 3 simultaneously active users at a SNR of $4 \mathrm{~dB}$.

Figs. 5, 6 and 7 present the results based on the BS as a transmitter and three nodes as receivers. In the presented scheme, each one of these nodes will be a receiver in the first time slot and a relay node in the second time slot. These figures illustrate that the performance of the cooperative OSIC scheme for downlink channels has an equal value of SNR (from $-10 \mathrm{~dB}$ to $10 \mathrm{~dB}$ ), while the SNR of the inter-user channel equals $15 \mathrm{~dB}$. 
Note that the results of this diversity are the outcome of cooperation between the users to share some results of OSIC detection. Another observation is the results of the cooperative OSIC scheme, which is indicated by the curve between the bit error rate (BER) in relation to SNR. These users must be able to identify a suitable partner to obtain optimal performance through their knowledge of the inter-user channel characteristics between each user and its' partner.

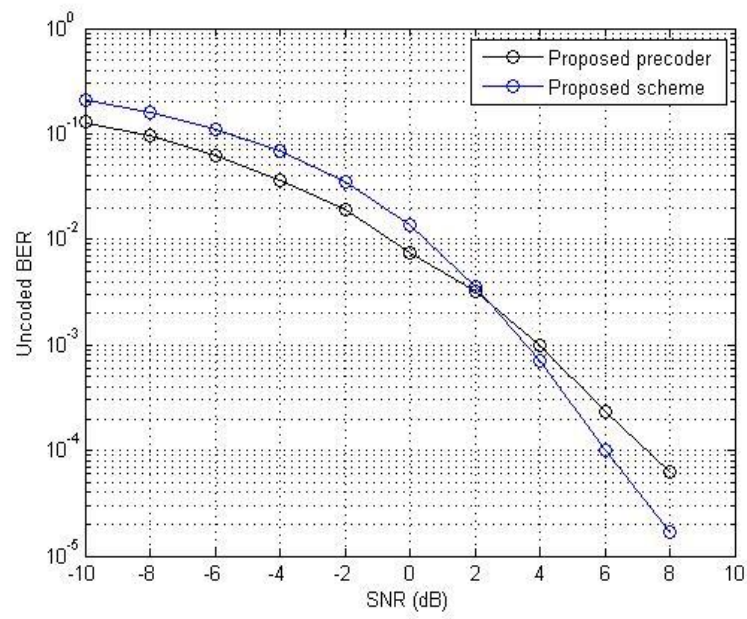

Fig.5 MU-MIMO system for $M=6, U=3, N i=3$, Rician inter user channel with $K=15 \mathrm{~dB}$ and $\beta=0.15$.

As seen in Fig.5, in the case of the proposed scheme, the performance of the system gradually and continuously improved with increases in the values of SNRs. On the other hand, in the case of the proposed precoder, the performance of the system was dramatically improved at significantly lower values of SNRs.

Unfortunately, when the values of SNRs increased, performance improvement slowly stabilized even at high values of SNRs comparing to the proposed scheme.

That is because of noise and inter-user interference factors. For the proposed precoder, which supports inter-user interference but does not benefit from it, the system performed better than the proposed scheme at low SNR values because the effect of noise was bigger than the effect of inter-user interference. Therefore, at low SNR values, the major factor limiting the performance of the system was the noise.

On the other hand, the proposed scheme, which supports multi-user interference to take advantage of it, outperformed the proposed precoder at high values of SNRs because the effect of inter-user interference as the useful signal was greater than the effect of noise on the system. Therefore, at high values of SNRs, the major factor limiting the performance of the system was inter-user interference.

Fig. 6 illustrates the comparison of the proposed precoder and the proposed scheme to select the optimum value of $\beta$. In the proposed scheme, two scenarios are observed compared to the proposed precoder. In the first scenario, when $\beta=0.15$, the proposed precoder has better performance than the proposed scheme for SNR values lower than $3 \mathrm{~dB}$.

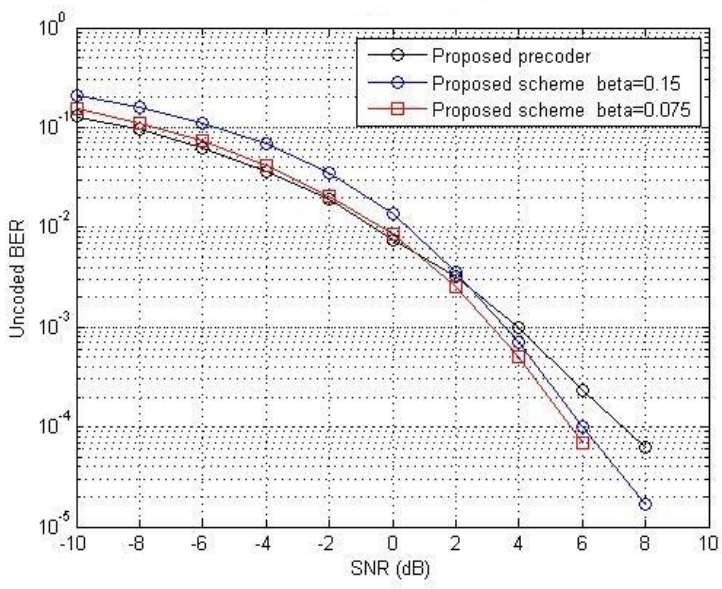

Fig.6 MU-MIMO system for $M=6, U=3$ and $N i=3$, Rician inter user channel with $K=15 \mathrm{~dB}$.

This result is attributed to the high effect of noise and MU interference in the second time slot of the proposed scheme when the users will share the signals with high interference value. In the second scenario, when $\beta=0.075$, the effect of the interference on sharing signals is reduced.

Therefore, the performance of the proposed scheme is improved when SNR $>0 \mathrm{~dB}$. Therefore, the interference is the main factor limiting the capacity of the system because the proposed scheme turns the interference signals into useful signals when it detects these signals using OSIC. Furthermore, it can benefit from the MU interference by sharing these signals among users. Therefore, the proposed scheme with $\beta=$ 0.075 enjoys better performance than the proposed scheme with $\beta=0.15$.

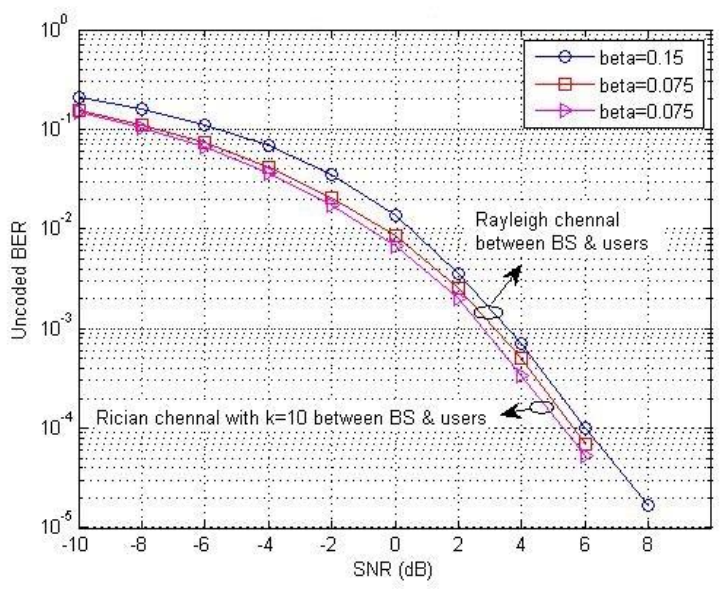

Fig.7 MU-MIMO system for $M=6, U=3, N i=3$, Rician channel between users with $K=15 \mathrm{~dB}$

Fig.7 shows the BER results for different system configurations. Although the performance of the proposed scheme significantly improved when the channel between BS and users is a Rician channel with $K=10 \mathrm{~dB}$. 


\section{CONCLUSION}

This study proposed a novel MU-MIMO downlink scheme that appropriately combines an SLR-based beamforming precoding scheme (with different power constraints) and an OSIC-MU detection scheme to improve the performance of the MU-MIMO system.

To achieve the OSIC-MU detection of the proposed scheme, we also presented a Hybrid Relaying model of AAF and DAF cooperative algorithm to share the specific results of OSIC detection in each user. The signal that leaked from the original signal was reintroduced to its real destination to take advantage of it. In the proposed scheme, the complexity in the BS will be reduced by reducing the design procedure for beamforming precoding. Because in the conventional SLRbeamforming scheme the precoder procedure needs to the proportionality constant. The proportionality constant is chosen to normalize the norm of the precoder to be unity. Comparing this solution to the proposed scheme; there is no need for this step in BS.

On the other hand, the Hybrid Relaying cooperative SLR transmission scheme did not use coding in the BS and thus not using the decoding and recoding in the relay without increasing the effect of noise. In this way, the complexity of BS will be reduced. Also, the processing latency will be reduced, thus reducing the complexity load in real-time detection in the relay. Simulations were carried out over the Rayleigh fading downlink channel in the first time slot and then over the Rician fading inter-user channel in the second time slot.

Simulation results demonstrated that the proposed scheme outperforms the classical SLR-based beamforming precoding schemes. The performance of the proposed scheme with different numbers of users and numbers of antennas was also investigated. The critical parameters of the proposed scheme were likewise demonstrated.

\section{CONTRIBUTION OF THE WORK}

The contribution of this paper is exploiting the leakage power of MU MIMO Beamforming, by applying the following model and scheme:

1-This study proposed a cooperative model for the downlink MU-MIMO Beamforming system.

2-This paper considers an alternative approach based on the concept of SLR to find a beamforming weight that can improve the OSIC performance of the MIMO scheme without significantly increasing the complexity of the system.

\section{REFERENCES}

[1] E. Telatar, "Capacity of multi-antenna Gaussian channels," Euro. Trans. Telecommun., vol. 10, no. 6, pp. 585-595, November 1999. DOI: $10.1002 /$ ett.4460100604.

[2] Q. H. Spencer, C. B. Peel, A.L. Swindlehurst, and M. Haardt, "An Introduction to the Multi-user MIMO Downlink," IEEE Commun. Mag., vol. 24, no. 10, pp. 60-67, October 2004. DOI: 10.1109/ MCOM. 2004.1341262.
[3] P. Viswanath and D. Tse, "Sum capacity of the vector Gaussian broadcast channel and uplink-downlink duality," IEEE Trans. Inform. Theory, vol. 49, no. 8, pp. 1912-1921, August 2003.

DOI: 10.1109/TIT.2003.814483.

[4] W. Yu and J.Cioffi, "Sum capacity of Gaussian vector broadcast channels," IEEE Trans. Inform. Theory, vol. 50, no. 9, pp. 1875-1892, September 2004. DOI: 10.1109/TIT.2004.833336.

[5] F. Boccardi, R. W. Heath, A. Lozano, T. L. Marzetta, and P. Popovski, "Five disruptive technology directions for 5G," IEEE Commun. Mag., vol. 52, no. 2, pp. 74-80, Feb. 2014. DOI: 10.1109/MCOM. 2014.6736746.

[6] M. Vu and A. Paulraj, "MIMO wireless linear precoding," IEEE Signal Processing Magazine, vol. 24, no. 5, pp. 86-105, Sept. 2007. DOI: 10.1109/MSP.2007.904811.

[7] E. L. Bengtsson, F. Rusek, S. Malkowsky, F. Tufvesson, P. C. Karlsson, and O. Edfors, "A simulation framework for multiple-antenna terminals in 5G massive MIMO systems," IEEE Access, vol. 5, no. 99, pp. 26819 26831, 2017. DOI: 10.1109/ACCESS.2017.2775210.

[8] A. Gupta and R. K. Jha, "A survey of 5G network: Architecture and emerging technologies," IEEE Access, vol. 3, pp. 1206-1232, 2015. DOI: 10.1109/ACCESS.2015.2461602.

[9] Cong Shen, "Downlink Multi-User MIMO Precoding Design Via SignalOver-Leakage Capacity,” IEEE Access, vol. 6, pp. 2812-2824, Dec. 2017. DOI: 10.1109/ACCESS.2017.2785347.

[10] A. Tarighat, M. Sadek, and A. H. Sayed, "A multiuser beamforming scheme for MIMO channels based on maximizing signal-to-leakage ratios," IEEE International Conference on Acoustics, Speech, and Signal Processing, pp. 1129-1132, March 2005.

DOI: 10.1109/ICASSP.2005.1415913.

[11] C. ÇiFTLiKLi, M. AL-OBAIDI, "Combining Alamouti STBC with Block Diagonalization for Downlink MU-MIMO System over Rician Channel for 5G," Infocommunications Journal, vol. XI, no. 1, pp. 22-28, March 2019.

[12] H. Du and P. J. Chung, "A probabilistic approach for robust leakagebased MU-MIMO downlink beamforming with imperfect channel state information," IEEE Trans. Wireless Commun., vol. 11, no. 3, pp. 12391247, Mar. 2012. DOI: 10.1109/TWC.2012.011012.111260.

[13] G. J. Foschini and M. J. Gans, "On limits of wireless communications in a fading environment when using multiple antennas," Wireless Pers. Commun., vol. 6, pp. 311-335, March 1998. DOI: $10.1023 /$ A: 1008889222784 .

[14] Q. H. Spencer, A. L. Swindlehurst, and M. Haardt, "Zeroforcing methods for downlink spatial multiplexing in multiuser MIMO channels," IEEE Transactions on Signal Processing, vol. 52, pp. 461-471, Feb. 2004. DOI: 10.1109/TSP.2003.821107.

[15] M. Bengtsson, "A pragmatic approach to multi-user spatial multiplexing," IEEE Sensor Array and Multichannel Signal Processing Workshop, Rosslyn, VA, pp. 130-134, Aug. 2002. DOI: 10.1109/SAM. 2002.1191014

[16] R. Chen, J. G. Andrews, and R. W. Heath, "Multiuser space-time block coded MIMO with downlink precoding," IEEE International Conference on Communications, Paris, France, pp. 2689-2693, June 2004. DOI: 10.1109/ICC.2004.1313019.

[17] K. Wong, R. D. Murch, and K. B. Letaief, "Performance enhancement of multiuser MIMO wireless communication systems," IEEE Transactions on Communications, vol. 50, pp. 1960-1970, Dec. 2002. DOI: 10.1109/TCOMM.2002.806503.

[18] H. Liu, X. Cheng, Z. Zhou, G. Wu, "Block Diagonalization Eigenvalue Based Beamforming precoding design for Downlink Capacity Improvement in Multiuser MIMO channel," IEEE International Conference on Wireless Communications and Signal Processing (WCSP), November 2010. DOI: 10.1109/WCSP.2010.5633549.

[19] Y. Jiang, J. Li, and W. W. Hager, "Joint Transceiver Design for MIMO communications using Geometric Mean Decomposition," IEEE Trans. Signal Process., vol. 53, no. 10, pp. 3791-3803, October 2005. DOI: 10.1109/TSP.2005.855398.

[20] Y. S. Cho, J. Kim, W. Y. Yang, C. G. Kang, "Wireless Communications with MATLAB," John Wiley \& Sons, New York, p.544, 25 Aug 2010. 
[21] J. N. Laneman and G. W. Wornell, "Cooperative diversity in wireless networks: efficient protocols and outage behavior," IEEE Trans. Inform. Theory, vol. 50, no. 12, pp. 3062-3080, 2004. DOI: 10.1109/TIT .2004.838089

[22] A. Sendonaris, E. Erkip, and B. Aazhang, "User Cooperation Diversity Part I and Part II," IEEE Trans. Commun., vol. 51, no. 11, pp. 1927-1948, Nov. 2003. DOI: 10.1109/TCOMM.2003.819238.

[23] E. Bjornson, M. Bengtsson, B. Ottersten, "Optimal multiuser transmit beamforming: A difficult problem with a simple solution structure," IEEE Signal Process. Mag., vol. 31, no. 4, pp. 142-148, Jul. 2014. DOI: 10.1109/MSP.2014.2312183.

[24] X. Shi, C. Siriteanu, S. Yoshizawa, Y. Miyanaga, "Realistic Rician Fading Channel based Optimal Linear MIMO Precoding Evaluation," IEEE International Symposium on Communications Control and Signal Processing (ISCCSP), 2012. DOI: 10.1109/ISCCSP.2012.6217780.

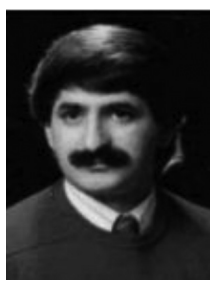

Cebrail ÇiFTLiKLi was born in K. Maras, Turkey, in 1961. He received the Ph.D. degree in electronics engineering from Erciyes University in 1990. In 2004, he joined Erciyes University Kayseri Vocational College as Professor where he is now principal. Prof. Dr. Ciftlikli's current research interests include spread spectrum communications, wireless ATM/LAN, signal processing, DSCDMA system engineering, RF power amplifier linearization for wireless communication systems. He works at Department of Electronics and Automation, Kayseri University, Kayseri, Turkey.

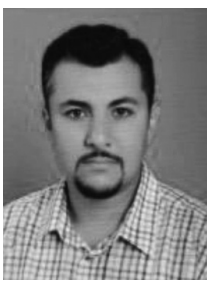

Musaab AL-OBAIDI was born in Bagdad, Iraq, in 1981. 2000-2004 B.Sc. in Department of Electrical Engineering, Al-Mustanseria University, Iraq. 20102013 M.Sc. in Electrical Engineering, Universiti Tenaga Nasional, Malaysia. Since 2014 he is Ph.D. student in Department of Electrical and Electronics Engineering, Erciyes University, Kayseri, Turkey.

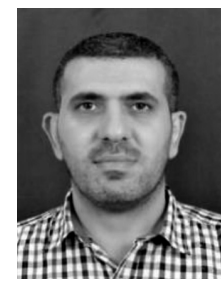

Mohammed Fadhil received the B.Sc. degree in electrical engineering from AL-Mustansiria University, College of Engineering, Iraq in 2001 and the M.Sc. degree from University Tenaga National (UNITEN), Malaysia in 2013. He is currently Ph.D. candidate at Universiti Kebangsaan Malaysia (UKM), Kuala Lumpur, Malaysia. His research interests include wireless communication, with a focus on cooperative communication and beamforming.

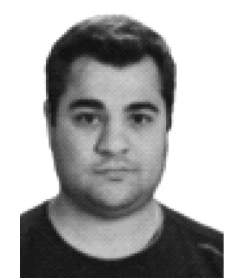

Wael AL-OBAIDI was born in Bagdad, Iraq, in 1985. 2003-2007 B.Sc. in Department of Electrical Engineering, University of Technology (UOT), Iraq. $\mathrm{He}$ is currently M.Sc. candidate at Electrical and Electronics Engineering, Erciyes University, Kayseri, Turkey. 